\title{
Transcription Systems
}

\section{Arabic Script}

Diacritical signs have been avoided in all transcriptions proposed in the CENTRAL EURASIAN READER. For the Arabic script, for this first issue of the journal the distinct vocalisation systems of Iranian and Dari or Tajik Persian have been preserved in the transcription, as well as the varying value of certain consonants (for instance that of the Arabic character 9 being transcribed, as a consonant, respectively, by a " $v$ " or by a "w").

\begin{tabular}{|c|c|c|c|}
\hline Arabic character & $\begin{array}{c}\text { Latin } \\
\text { Transcription }\end{array}$ & Arabic character & $\begin{array}{c}\text { Latin } \\
\text { transcription }\end{array}$ \\
\hline$i$ & a & ص & s \\
\hline 1 & a / e or i / o or u & ض & $\mathrm{z}$ \\
\hline 1 & $o / u$ & $b$ & $\mathrm{t}$ \\
\hline ب & b & b & $\mathrm{z}$ \\
\hline ب ب ب & $\mathrm{p}$ & $\varepsilon$ & ‘ \\
\hline$ت$ & $\mathrm{t}$ & $\dot{\varepsilon}$ & gh \\
\hline ث & th & ف & $\mathrm{f}$ \\
\hline ج & $\mathrm{j}$ & ق & $\mathrm{q}$ \\
\hline ङ & $\mathrm{ch}$ & ك & $k$ \\
\hline$\tau$ & $\mathrm{h}$ & 3 & $\mathrm{~g}$ \\
\hline$\dot{\tau}$ & $\mathrm{kh}$ & s] & ng \\
\hline د & $\mathrm{d}$ & J & 1 \\
\hline j & $\mathrm{dh}$ & s & $\mathrm{m}$ \\
\hline J & $\mathrm{r}$ & ن & $\mathrm{n}$ \\
\hline j & $\mathrm{z}$ & و & $\mathrm{w}$ or $\mathrm{v} / \mathrm{u}$ or ow \\
\hline$j$ & $\mathrm{zh}$ & 。 & $\mathrm{h}$ \\
\hline س س & $\mathrm{s}$ & ى (- مى & $\mathrm{y} / \mathrm{i}$ \\
\hline 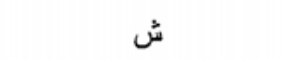 & $\mathrm{sh}$ & 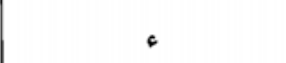 & , \\
\hline
\end{tabular}


Arabic Script Reformed (Modern Uighur)

\begin{tabular}{|c|c|c|c|}
\hline Arabic character & $\begin{array}{l}\text { Latin } \\
\text { Transcription }\end{array}$ & Arabic character & $\begin{array}{c}\text { Latin } \\
\text { transcription }\end{array}$ \\
\hline نا & A a & ق & Q q \\
\hline نه & $\ddot{A}$ ä & s & $\mathrm{K} \mathrm{k}$ \\
\hline ب & B b & 3 & $\mathrm{Gg}$ \\
\hline ب & P p & ك & $\mathrm{ng}$ \\
\hline ت & $\mathrm{Tt}$ & $J$ & I. 1 \\
\hline ج & $\mathrm{Jj}$ & 5 & $\mathrm{M} \mathrm{m}$ \\
\hline ६ & $\mathrm{Ch} \mathrm{ch}$ & ن & $\mathrm{N} \mathrm{n}$ \\
\hline$\dot{\tau}$ & Kh kh & $\Delta$ & $\mathrm{H} \mathrm{h}$ \\
\hline د & D d & نو & $0 \circ$ \\
\hline s & $\mathrm{R} \mathrm{r}$ & نو & $\mathrm{U} \mathrm{u}$ \\
\hline j & $\mathrm{z} \mathrm{z}$ & نو" & ö ö \\
\hline$j$ & $\mathrm{zh} \mathrm{zh}$ & نو & $\ddot{U} \ddot{u}$ \\
\hline س & $\mathrm{S} \mathrm{s}$ & $\dot{3}$ & $\mathrm{w} w$ \\
\hline ش & Sh sh & ني & $\mathrm{E} \mathrm{e}$ \\
\hline$\dot{\varepsilon}$ & Gh gh & نى & $\mathrm{Ii}$ \\
\hline ن & $\mathrm{Ff}$ & ي & Y y \\
\hline
\end{tabular}

Armenian Script

\begin{tabular}{|c|c|c|c|}
\hline$u w$ & $\mathrm{~A} a$ & $\cup \cup$ & $\mathrm{M} \mathrm{m}$ \\
\hline$f F$ & $\mathrm{~B} \mathrm{~b}$ & $3 \mathrm{~J}$ & $\mathrm{Y} \mathrm{y} / \mathrm{H} \mathrm{h}$ \\
\hline 99 & $G g$ & ᄂ u & $\mathrm{N} \mathrm{n}$ \\
\hline $7 n$ & D d & $\subset 2$ & Sh sh \\
\hline$t \quad t$ & $\mathrm{E} \mathrm{e} / \mathrm{Ye} \mathrm{ye}$ & $n n$ & $\mathrm{O} \mathrm{o} / \mathrm{Vo}$ vo \\
\hline $2 q$ & Z z & $2 \varepsilon$ & $\mathrm{Ch} \mathrm{ch}$ \\
\hline t $t$ & E e & $\eta \mu$ & $\mathrm{P} p$ \\
\hline$C \mathrm{D}$ & E e & 22 & $\mathrm{~J} \mathbf{j}$ \\
\hline$\theta \mathrm{P}$ & $\mathrm{T} \mathrm{t}$ & $n n$ & $\mathrm{R} \mathrm{r}$ \\
\hline d d & $\mathrm{Zh} \mathrm{zh}$ & $\mathrm{U} u$ & $\mathrm{~S} \mathrm{~s}$ \\
\hline h $\mathrm{h}$ & I i & 44 & $\mathrm{~V} v$ \\
\hline L L & L l & $S$ un & $\mathrm{T} \mathrm{t}$ \\
\hline to tu & $\mathrm{Kh} \mathrm{kh}$ & $r n$ & $\mathrm{R} \mathrm{r}$ \\
\hline 00 & $\mathrm{Tz} \mathrm{tz}$ & $3 \mathrm{~g}$ & Ts ts \\
\hline
\end{tabular}




\begin{tabular}{|c|c|c|c|}
\hline 44 & K k & $\vdash L$ & $\mathrm{~V} v$ \\
\hline $\mathrm{h}$ & $\mathrm{H} \mathrm{h}$ & (1) ये & $\mathrm{Pp}$ \\
\hline 28 & $\mathrm{Dz} \mathrm{dz}$ & $2 p$ & Q q \\
\hline$\eta \eta$ & Gh gh & 00 & 00 \\
\hline 66 & $\mathrm{Ch} \mathrm{ch}$ & $\$ \$$ & $\mathrm{~F} \mathrm{f}$ \\
\hline
\end{tabular}

\section{Cyrillic Script}

\begin{tabular}{|c|c|c|c|}
\hline $\begin{array}{l}\text { Cyrillic } \\
\text { character }\end{array}$ & $\begin{array}{c}\text { Latin } \\
\text { Transcription }\end{array}$ & $\begin{array}{l}\text { Cyrillic } \\
\text { character }\end{array}$ & $\begin{array}{c}\text { Latin } \\
\text { transcription }\end{array}$ \\
\hline $\mathrm{A}$ a & A a & $\mathrm{P} p$ & $\mathrm{R} \mathrm{r}$ \\
\hline Б 6 & $\mathrm{~B} \mathrm{~b}$ & $\mathrm{P} p$ & $\mathrm{R} \mathrm{r}$ \\
\hline B B & $\mathrm{V} v$ & $\mathrm{CC}$ & $\mathrm{S} \mathrm{s}$ \\
\hline$\Gamma \mathrm{r}$ & $\mathrm{G} \mathrm{g}$ & $\mathrm{T} \mathrm{T}$ & $\mathrm{Tt}$ \\
\hline$f_{F}$ & Gh gh & $\mathrm{y} y$ & $\mathrm{Uu}$ \\
\hline д д & D d & y̆ y̆ & $\mathrm{U} u$ \\
\hline $\mathrm{E} \mathrm{e}$ & $\mathrm{E} \mathrm{e}$ & $¥ ¥$ & $\mathrm{U} u$ \\
\hline Ë ë & $\mathrm{E} \mathrm{e}$ & $\mathrm{Y} Y$ & $\mathrm{U} u$ \\
\hline$\partial$ ә & $\mathrm{E} \mathrm{e}$ & $\Phi \phi$ & F f \\
\hline Ж ж & $\mathrm{Zh} \mathrm{zh}$ & $\mathrm{Xx}$ & $\mathrm{Kh} \mathrm{kh}$ \\
\hline Ж ж & $\mathrm{J} \mathrm{j}$ & $X \times$ & $\mathrm{H} \mathrm{h}$ \\
\hline 33 & $\mathrm{Zz}$ & $\mathrm{h} \mathrm{h}$ & $\mathrm{H} \mathrm{h}$ \\
\hline И и & $\mathrm{li}$ & ц ц & T's ts \\
\hline Й й & $\mathrm{I} \mathrm{i}$ & ૫ ૫ & $\mathrm{Ch} \mathrm{ch}$ \\
\hline K к & K k & 44 & $\mathrm{~J} \mathrm{j}$ \\
\hline Қ к & Q q & III ш & Sh sh \\
\hline $\mathrm{K} K$ & $\mathrm{Q} \mathrm{q}$ & щ щ & Shch shch \\
\hline Л $\pi$ & L 1 & b & ' \\
\hline $\mathrm{M} \mathrm{M}$ & $\mathrm{M} \mathrm{m}$ & Ы ы & Y y \\
\hline $\mathrm{H} \mathrm{H}$ & $\mathrm{N} \mathrm{n}$ & $\mathrm{b} \mathrm{b}$ & \\
\hline Н н & $\mathrm{Ng} n g$ & Э э & $\mathrm{E} \mathrm{e}$ \\
\hline 00 & 00 & Ю ю & Iu iu \\
\hline$\Pi \pi$ & $\mathrm{P} p$ & Я я & la ia \\
\hline$\theta \theta$ & 00 & & \\
\hline
\end{tabular}

\section{Hebraic Script}

$\begin{array}{ll}\text { a } & \text { A a } \\ \text { B } & \text { B b } \\ \text { g } & \text { G g } \\ \text { D } & \text { D d } \\ \text { T } & \text { H h } \\ \text { W } & \text { W w } \\ \text { Z } & \text { Z z }\end{array}$




\begin{tabular}{cc|cc}
$\mathrm{h}$ & $\mathrm{H} \mathrm{h}$ & $\mathrm{q}$ & $\mathrm{K} \mathrm{k}$ \\
$\mathrm{f}$ & $\mathrm{T} \mathrm{t}$ & $\mathrm{r}$ & $\mathrm{R} \mathrm{r}$ \\
$\mathrm{y}$ & $\mathrm{J} \mathrm{j}$ & $\mathrm{C}$ & $\mathrm{S} \mathrm{s}$ \\
$\mathrm{d}$ & $\mathrm{K} \mathrm{k}$ & $\mathrm{h}$ & $\mathrm{T} \mathrm{t}$ \\
$\mathrm{k}$ & $\mathrm{K} \mathrm{k}$ & &
\end{tabular}

\section{Ideographic Scripts}

As far as Japanese in concerned, a simplified version of the classical, English-centred Hepburn system has been given preference here for more easiness-especially for facilitating the journal's edition on the internet, without deforming the most current transcriptions-: e.g., 中央アジア史: Chuo Ajia shi (“History of Middle Asia"); あるジ ヤヂーどの肖像: aru jadiido no shozo ("portrait of a Jadid").

The transcription adopted for Chinese language is that of the pinyin system. 
Qui veut écrire une œuvre qui soit "toutes choses" pour d'autres êtres, doit s'isoler de sa propre vie, se bâtir en dehors d'elle une solitude, rassembler toutes les forces de son esprit et de son cœur et par delà le temps construire ou conquérir de la durée; une œuvre, c'est d'abord une désobéissance, et presque une provocation.

Armand Robin Dissertation sur Mme de Sévigné, Concours d'agrégation 1936 